\title{
Anti-inflammatory and anti-oxidative effects of 3-(naphthalen-2-yl(propoxy)methyl)azetidine hydrochloride on $\beta$-amyloid-induced microglial activation
}

\author{
Seung-Ju Yang ${ }^{1}$, Jiae Kim ${ }^{2}$, Sang Eun Lee ${ }^{2}$, Jee-Yin Ahn ${ }^{3}$, Soo Young Choi ${ }^{4}$ E Sung-Woo Cho ${ }^{2, *}$ \\ ${ }^{1}$ Department of Biomedical Laboratory Science, Konyang University, Daejeon 35365, ${ }^{2}$ Department of Biochemistry and Molecular Biology, \\ University of Ulsan College of Medicine, Seoul 05505, ${ }^{3}$ Department of Molecular Cell Biology, Center for Molecular Medicine, Samsung \\ Biomedical Research Institute, Sungkyunkwan University School of Medicine, Suwon 16419, ${ }^{4}$ Department of Biomedical Science and \\ Research Institute for Bioscience and Biotechnology, Hallym University, Chunchon 24252, Korea
}

\begin{abstract}
We aimed to assess the anti-inflammatory and antioxidative properties of KHG26792, a novel azetidine derivative, in amyloid $\beta$ (A $\beta$ )-treated primary microglial cells. KHG26792 attenuated the $A \beta$-induced production of inflammatory mediators such as IL-6, IL-1 $\beta$, TNF- $\alpha$, and nitric oxide. The levels of protein oxidation, lipid peroxidation, ROS, and NADHP oxidase enhanced by $A \beta$ were also downregulated by KHG26792 treatment. The effects of KHG26792 against the A $\beta$-induced increases in inflammatory cytokine levels and oxidative stress were achieved by increasing the phosphorylation of Akt/ GSK-3 $\beta$ signaling and by decreasing the $A \beta$-induced translocation of NF- $\mathrm{kB}$. Our results provide novel insights into the use of KHG26792 as a potential agent against $A \beta$ toxicity, including its role in the reduction of inflammation and oxidative stress. Nevertheless, further investigations of cellular signaling are required to clarify the in vivo effects of KHG26792 against A $\beta$-induced toxicity. [BMB Reports 2017; 50(12): 634-639]
\end{abstract}

\section{INTRODUCTION}

Amyloid beta $(A \beta)$ peptide accumulation and neurofibrillary tangle formation are major events in the pathology of Alzheimer's disease (AD) (1). A $\beta$ deposition activates microglia and triggers the release of proinflammatory cytokines such as interleukin-1 beta (IL-1 $\beta$ ) and tumor necrosis factor alpha (TNF- $\alpha$ ), which induce neuroinflammation and neuronal

${ }^{*}$ Corresponding author. Tel: +82-2-3010-4278; Fax: +82-2-30104278; E-mail: swcho@amc.seoul.kr

https://doi.org/10.5483/BMBRep.2017.50.12.189

Received 22 September 2017, Revised 7 October 2017, Accepted 12 October 2017

Keywords: Amyloid $\beta$, Inflammation, N-Adamantyl-4-methylthiazol2-amine, Oxidative stress degeneration in the brain (2). The suppression of glial activation attenuated synaptic dysfunction in a mouse model of $\mathrm{AD}$ and activated microglia surrounding neuritic plaques have been found to continuously release pro-inflammatory mediators (3). Thus, microglia-mediated neuroinflammation is one of the key characteristics of $A D$. A $\beta$-induced microglial activation also leads to the secretion of cytotoxic factors such as reactive oxygen species (ROS) and nitric oxide (NO), which can cause neuronal cell damage and contribute to the progression of $A D$ (4). The p38 signaling pathway and the transcription factor nuclear factor kappa $\mathrm{B}(\mathrm{NF}-\mathrm{\kappa B})$ play an important role in the regulation and activity of pro-inflammatory cytokines in microglial cells (5). Hence, regulatory substances that attenuate $A \beta$-induced microglial activation may be a suitable target when developing drugs for $A D$.

To identify more effective drugs against $A \beta$-induced cell death, we synthesized and characterized azetidine derivatives, which have been suggested to be useful therapeutic agents for several diseases (6). For instance, previous studies described the memory-restorative effect of ezetimibe-another well-known azetidine derivative-in memory dysfunctions associated with dementia of Alzheimer's type, and discussed the potentially beneficial action of ezetimibe in suppressing plaque inflammation (7). The biological functions of azetidine derivatives against serotonin, norepinephrine, and dopamine transporters have also been reported $(8,9)$. Recently, we described the protective effects of KHG26792-a novel azetidine derivativeon the ATP-induced activation of the NFAT and MAPK pathways through the P2X7 receptor in BV-2 cells and on hypoxia-induced toxicity through the suppression of microglial activation in BV-2 cells $(10,11)$. Nevertheless, the mechanism responsible for the action of KHG26792 remains unclear.

In our current study, we investigated the mechanism and antioxidative and anti-inflammatory potential of KHG26792 in primary microglial cells to evaluate the efficacy of KHG26792 against $A \beta$-induced microglial activation. 


\section{RESULTS AND DISCUSSION}

\section{KHG26792 attenuates the $A \beta$-induced toxicity in primary microglial cells}

Unregulated neuroinflammatory responses following exposure to $A \beta$ in activated microglia may induce neurodegeneration and the production of various pro-inflammatory cytokines that cause neurotoxicity in neighboring neurons (12). Hence, the regulation of microglia-meditated inflammation may be a suitable novel therapeutic approach for the treatment of neurodegenerative diseases such as $\mathrm{AD}$ (13). In the present study, we aimed to clarify the anti-inflammatory effects of KHG26792 on $A \beta$-induced microglial activation in rat primary microglia cells, and the possible underlying mechanisms.

When microglial cells were incubated with $A \beta$, cell viability was decreased (Fig. 1A) and LDH leakage was increased (Fig. 1B). However, treatment with $50 \mu \mathrm{M}$ of KHG26792 significantly attenuated the percentage of both cell viability and LDH leakage as compared with the A $\beta$-treated control. KHG26792 alone did not affect any appreciable toxicity and the viability of microglial cells treated with KHG26792 alone approached to almost $100 \%$ of the control (Fig. 1). Based on these results, $50 \mu \mathrm{M}$ KHG26792 was selected as an optimal KHG26792 concentration for subsequent experiments.

KHG26792 attenuates the $A \beta$-induced production of inflammatory molecules in primary microglial cells

Next, we investigated the effects of KHG26792 on the A $\beta$-induced production of inflammatory molecules in primary microglial cells. The production of IL- 6, IL-1 $\beta$ and TNF- $\alpha$ was markedly increased by $A \beta$, although KHG26792 attenuated the $A \beta$-induced levels of pro-inflammatory cytokines (Fig. $2 \mathrm{~A}-\mathrm{C})$. A $\beta$ treatment also increased $\mathrm{NO}$ production, compared with the untreated controls (Fig. 2D). NO-a reactive nitrogen intermediate - plays an important role in cellular signaling pathways and can mediate many pathophysiological processes (14). However, the NO levels significantly decreased in the
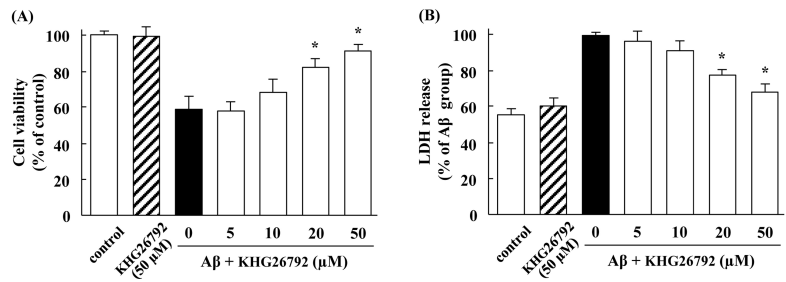

Fig. 1. Protective effects of KHG26792 in the $A \beta$-induced primary microglial cells. Cells were incubated with $50 \mu \mathrm{M} A \beta$ for 2 h, followed by various concentrations of KHG26792 for 24 h. MTT assay (B) and LDH assay (C) and was assessed as described in MATERIALS AND METHODS. The resulting values represent the mean \pm SD of 3 independent experiments. *Indicates statistical significance between $A \beta$ group and $A \beta$ group treated with KHG26792 $(P<0.01)$.
KHG26792-treated groups, as compared with the corresponding A $\beta$-treated groups (Fig. 2D). Moreover, treatment with KHG26792 alone did not lead to any significant effects on inflammatory molecules in cultured microglia cells (Fig. 2).

Although our results showed that KHG26792 successfully inhibits the levels of cytokines, we can't completely exclude the possibility that the effects of KHG26792 may be due to the reduction of $A \beta$-induced cytotoxicity. This remains for the future study.

\section{Anti-oxidative effects of KHG26792 in A $\beta$-induced primary microglial cells}

$A D$ is a well characterized disease and wherein $A \beta$-stimulated oxidative stress is closely related to $A D$ pathogenesis and progression, where $A \beta$ induces an increase in protein oxidation and lipid peroxidation (15). Previous studies reported that protein oxidation levels are increased in the brains and ventricular cerebrospinal fluid of $A D$ patients (16). Consistent with these previous reports, our present results showed that $A \beta$ increased the level of protein carbonyl (Fig. 3A), whereas KHG26792 effectively attenuated the $A \beta$-induced protein oxidation. These results support the potential of KHG26792 in the regulation of $A \beta$-induced protein oxidation in primary microglia cells. Increased HNE level has been implicated as lipid peroxidation markers in experimental $A D$ models and in the brain or cerebrospinal fluid of AD patients (17). Compared with the control group, HNE level increased in the A $\beta$-treated group and were significantly downregulated by KHG26792 treatment (Fig. 3B).

ROS are formed as byproducts of various normal cellular
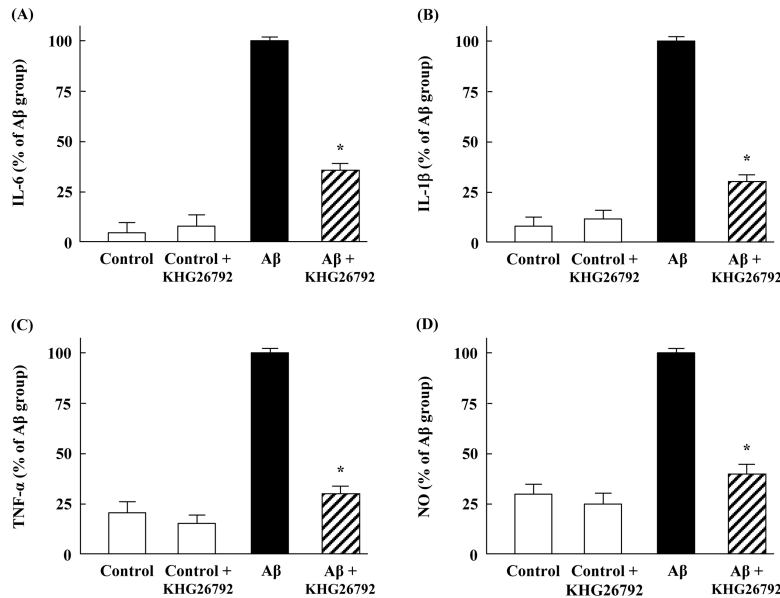

Fig. 2. Anti-inflammatory effects of $\mathrm{KHG} 26792$ in $\mathrm{A} \beta$-induced primary microglial cells. Cells were incubated with $50 \mu \mathrm{M} A \beta$ for $2 \mathrm{~h}$, followed by $50 \mu \mathrm{M}$ KHG26792 for $24 \mathrm{~h}$. The levels of IL-6 $(\mathrm{A})$, IL-1 $\beta$ (B), TNF- $\alpha(\mathrm{C})$, and NO (F) were assayed. The resulting values represent the mean \pm SD of 3 independent experiments. * Indicates statistical significance between $A \beta$ group and $A \beta$ group treated with KHG26792 ( $<<0.01)$. 

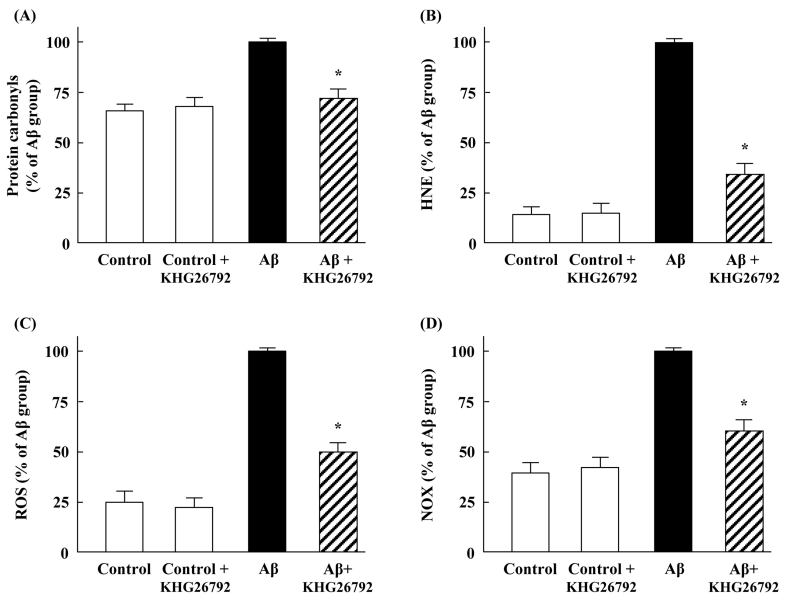

Fig. 3. Anti-oxidative effects of KHG26792 in $A \beta$-induced primary microglial cells. Cells were incubated with $50 \mu \mathrm{M} A \beta$ for $2 \mathrm{~h}$, followed by $50 \mu \mathrm{M}$ KHG26792 for $24 \mathrm{~h}$. The levels of protein carbonyls (A), HNE (B), ROS (C), and the activity of NOX (D) were assayed. The resulting values represent the mean $\pm \mathrm{SD}$ of 3 independent experiments. ${ }^{*}$ Indicates statistical significance between $A \beta$ group and $A \beta$ group treated with $K H G 26792(P<0.01)$.

processes involving interactions with oxygen, and increased ROS generation plays an important role in the pathological features of $A D$ (18). Moreover, $A \beta$ has been found to impair mitochondrial redox activity and induce ROS generation and oxidative stress during the early stages of AD pathogenesis, and this increased ROS formation may consequently lead to senile plaque accumulation in $A D$ brains (19). In the present study, we found that $A \beta$ increased ROS generation up to 4-fold, and that KHG26792 significantly attenuated the A $\beta$-induced ROS generation by $50 \%$ (Fig. $3 \mathrm{C}$ ), thus indicating that the anti-oxidative effects of KHG26792 against $A \beta$-induced oxidative stress could be due to its inhibition of excessive ROS generation by the $A \beta$ insult.

Previous studies have reported that the stimulation of NADPH oxidase increases the levels of superoxide and that $A \beta$ induces strong ROS production through NOX in BV2 microglial cells (20). Moreover, $A \beta$ increased the mRNA and protein expression levels of p47phox and gp91phox, along with the production of pro-inflammatory cytokines $(2,21)$. Therefore, we further examined the effect of KHG26792 on NOX activity in the $A \beta$-induced microglial cells. After exposure to $50 \mu \mathrm{M}$ $A \beta$, the NOX activity was enhanced up to 3-fold, relative to the control levels, whereas treatment with KHG26792 exerted a protective effect by lowering the NOX activity (Fig. 3D). Thus, our results suggest that KHG26792 reduces the A $\beta$-induced accumulation of ROS by regulating NOX, which is a source of $\mathrm{A} \beta$-induced ROS formation in primary microglial cells.
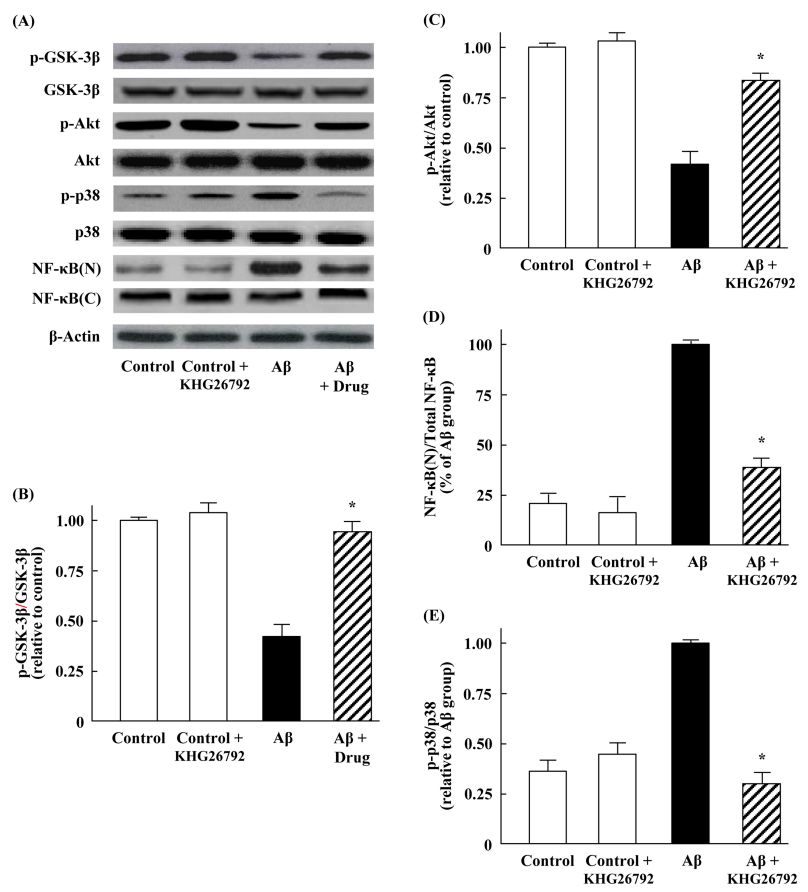

Fig. 4. Effects of KHG26792 on the protein expression of GSK3, Akt, and p38 in A $\beta$-induced primary microglial cells, as well as on the nuclear translocation of NF- $\kappa B$ p65. Primary microglial cells were incubated with $50 \mu \mathrm{M} A \beta$ for $2 \mathrm{~h}$, followed by 50 $\mu \mathrm{M}$ KHG26792 for $24 \mathrm{~h}$. Nuclear extracts were obtained from cell homogenates by using a nuclear extraction kit, according to the manufacturer's instructions (Sigma-Aldrich, St. Louis, MO). (A) Representative western blot images. (B-E) Relative expression levels were calculated using densitometry. The resulting values represent the mean $\pm \mathrm{SD}$ of 3 independent experiments. * Indicates statistical significance between $A \beta$ group and $A \beta$ group treated with KHG26792 $(\mathrm{P}<0.01)$.

Effects of KHG26792 on the protein expression of GSK3, Akt, $\mathrm{NF}-\mathrm{\kappa B}$, and $\mathrm{p} 38$ in $\mathrm{A} \beta$-induced primary microglial cells

To further explore the mechanisms underlying the antiinflammatory and anti-oxidative effects of KHG26792 in the primary microglial cells, we determined the alterations in the potential signal transduction pathway mediated by GSK3, Akt, p38, and NF-kB by using western blot analysis. Previous studies reported a direct or indirect relationship between GSK-3 $\beta$ and the neuropathology of AD (22). Furthermore, GSK-3 $\beta$ is part of the survival mechanism ensuring the control of $A \beta$-induced oxidative stress, and serves as a vital regulator in the inflammation responses of microglia (23). Moreover, the phosphorylation of GSK-3 $\beta$ plays an important role in the regulation of GSK-3 $\beta$ activity. Hence, it is worth evaluating whether the anti-oxidative effects of KHG26792 against ROS are mediated through the GSK-3 $\beta$ pathway. In the present study, we found that $A \beta$ did not affect the protein expression of GSK-3 3 , although $A \beta$ treatment reduced the expression of 
p-GSK-3 $\beta$ in primary microglial cells (Fig. 4A). However, treatment with KHG26792 significantly attenuated the protein level of p-GSK-3 $\beta$ close to the control levels (Fig. 4A, B).

Previous studies reported that $A k t / G S K-3 \beta$ is implicated in $A \beta$-induced toxicity. For instance, $A \beta$ inhibited the proliferation of neural stem cells by activating the PI3K/Akt/GSK-3 $\beta$ pathway, and the up-regulation of the AKT/GSK-3 $\beta$ pathway was correlated with the improvement of amyloid and tau pathologies in AD model mice (24). Consistent with these findings, our results showed that the phosphorylation of Akt was decreased in $A \beta$-treated cells. However, treatment with KHG26792 significantly attenuated the ratios of immunoreactivity for $\mathrm{p}$-Akt/Akt, when compared with the ratios in the A $\beta$-induced cells (Fig. 4A, C). These results suggest that increased inflammatory cytokine levels and oxidative stress caused by $A \beta$ are accompanied by the phosphorylation of Akt/GSK-3ß/p38, whereas the effects of KHG26792 against A $\beta$-induced toxicity in primary microglial cells are achieved by increasing the phosphorylation of Akt/GSK-3 $\beta$ signaling.

The activation of NF-kB has been observed in AD brains, and $A \beta$ stimulation can trigger NF- $\mathrm{KB}$ activation in neurons and glia (25). Therefore, the suppression of the NF- $\kappa B$ pathway in activated microglial cells may be a major target for anti-inflammatory disease treatment. Previous studies also suggested the possible involvement of the $A \beta / R O S / N F-\kappa B$ pathway in the progression of $A D$, because $A \beta$-induced ROS generation subsequently activates NF-kB (26). Upon activation, $\mathrm{NF}-\kappa \mathrm{B}$ is translocated from the cytosol to the nucleus. Therefore, we analyzed the cellular localization of NF- $\kappa B$ using western blot analysis. Our results showed that $A \beta$ induced the translocation of NF-kB from the cytosol to the nucleus in primary microglial cells (Fig. 4A, D), in agreement with the previous observations (26). However, KHG26792 treatment significantly decreased the nuclear expression of NF- $\mathrm{KB}$, with an attendant increase in the cytosolic expression of NF- $\mathrm{KB}$ (Fig. $4 A, D)$. These results indicate that the attenuation of $A \beta$-induced toxicity by KHG26792 may be related to a reduction in the activation of NF-kB.

Chronic exposure of human microglia to $A \beta$ can lead to an increase in p38 MAPK expression, and p38 MAPK activation has been considered to be involved in the pathogenesis of $A D$ (27). Our results showed that $A \beta$ induced an up-regulation of phosphorylated p-38 and that this up-regulation was effectively reduced by KHG26792 treatment (Fig. 4A, E). Hence, it is possible that the KHG26792-mediated inactivation of p38 and inhibition of NF-KB (Fig. 4) are associated with a reduced release of pro-inflammatory cytokines (Fig. 2) in $A \beta$-induced primary microglial cells. Thus, it is it is reasonable to speculate that the effects of KHG26792 against $A \beta$-induced toxicity may be related to the regulation of Akt/GSK-3 $\beta$ signaling as well as p38 and the NF-kB pathway, thereby enabling microglial cells to activate their antioxidant defense system.

Taken together, our findings suggest that KHG26792 may be a potential agent for treating $A \beta$-mediated toxicity. Nevertheless, further investigations of cellular signaling are required to clarify the in vivo effects of KHG26792 against A $\beta$-induced toxicity.

\section{MATERIALS AND METHODS}

\section{Materials}

$\mathrm{A} \beta_{25-35}$, phosphate-buffered saline (PBS), and dimethyl sulfoxide (DMSO) were obtained from Sigma-Aldrich (St. Louis, MO). Antibodies against GSK-3 $\beta$, p-GSK-3 $\beta$, Akt, p-Akt, p-38, p-p38, $\mathrm{NF}-\kappa \mathrm{B}$ p65, and $\beta$-actin were purchased from Cell Signaling Technology (Beverly, MA). 3-(Naphthalen-2-yl(propoxy)methyl) azetidine hydrochloride (KHG26792) was synthesized and purified as previously described (28). All other commercial reagents were of the highest available purity.

\section{Primary cultures of microglial cells and drug treatments}

All cell culture protocols were reviewed and approved by the Institutional Animal Care and Use Committee (IACUC) of the Asan Institute for Life Sciences, Asan Medical Center, which abides by the Institute of Laboratory Animal Resources (ILAR) guide. Primary rat microglial cells were prepared from the brains of embryonic day 16/17 Sprague-Dawley rats, and were digested by trypsin, followed by trituration as previously described, with a slight modification (3). In brief, after dissociation, homogenization, and resuspension, the mixed cells were cultured in DMEM/F-12 medium (Gibco, Grand Island, NY), supplemented with $10 \%$ fetal bovine serum, and maintained in a humidified incubator with $5 \% \mathrm{CO}_{2}$ at $37^{\circ} \mathrm{C}$. The media were changed twice a week. This procedure results in mixed glial cultures consisting of dividing astrocytes and microglial cells. After 14 days, microglia were separated from astrocytes by mild trypsinization and gentle shaking for $20 \mathrm{~min}$ at room temperature. Isolated microglial cells were plated into 24-well plates at a density of $2 \times 10^{5}$ cells/well, and the purity of microglia cultures was assessed using the CD11b antibody; more than $90 \%$ of cells were stained positively.

For drug treatment, cultured cells were incubated with 50 $\mu \mathrm{M} A \beta$ for $2 \mathrm{~h}$, followed by various concentrations of KHG26792 for 24 h. $A \beta_{25-35}$ was dissolved in sterile deionized and distilled water as a stock solution $(1 \mathrm{mM})$. Aliquots were incubated at $37^{\circ} \mathrm{C}$ for $72 \mathrm{~h}$, to promote the formation of aggregates (10). The $A \beta_{25-35}$ stock solution was diluted to the desired final concentrations in treatment medium. KHG26792 was freshly prepared as a stock solution $(10 \mathrm{mM})$ in DMSO, and then diluted to the desired final concentrations in treatment medium. Equivalent amounts of DMSO were used for controls and $A \beta$-treated cells throughout the entire experiment. LDH (lactate dehydrogenase) activity released from the cell was evaluated as an index of cellular toxicity. Briefly, cells were plated at a density of $5 \times 10^{5}$ cells in 96-well plates, and the extracellular and intracellular amounts of $\mathrm{LDH}$ were determined with the $\mathrm{LDH}$ toxicity detection kit from the Roche (Mannheim, Germany) according to the 
manufacturer's instructions. MTT assay was performed to determine cell viability by quantifying the color formation at $595 \mathrm{~nm}$ with an enzyme-linked immunosorbent assay (ELISA) microplate reader as described before (11).

\section{Measurement of IL- 6 , IL-1 $\beta$, TNF- $\alpha$, and NO}

TNF- $\alpha$, IL-6, and IL-1 $\beta$ levels were measured using commercial ELISA kits (R\&D Systems, Minneapolis, MN) according to the manufacturer's instructions, as previously described $(3,11)$. The optical density was detected with a microplate reader. The concentration of each sample was calculated from the linear equation derived from a standard curve of known concentrations of the cytokine. Samples of crude extracts with equal amounts of protein were used to determine the NO levels. Nitrite is a major product of $\mathrm{NO}$, and therefore, the concentration of $\mathrm{NO}$ in supernatants was measured from the amount of nitrite generated by using the Griess reagent (29). Optical density was measured at $540 \mathrm{~nm}$ by using a microplate reader (Molecular Devices Corp., Sunnyvale, CA).

\section{Measurement of protein oxidation, lipid peroxidation, ROS, and NOX}

The levels of protein carbonyls for protein oxidation were measured with the spectrophotometric method using an extinction coefficient of $22,000 \times 10^{6} \mathrm{nmol} / \mathrm{ml}$ for aliphatic hydrazones (30). The levels of HNE were measured by slot-blot analysis using the anti-HNE antibody raised in rabbits as the primary antibody (5:1,000 dilution) (31). The membrane was developed with nitro-blue tetrazolium chloride and 5-bromo-4-chloro-3'-indolyphosphate p-toluidine substrate.

ROS levels were measured using $2^{\prime}, 7^{\prime}$-dichlorofluorescin diacetate (DCF-DA), which is converted by ROS to fluorescent DCF (11). The concentrations of ROS were determined using the oxidation-sensitive probe DCF-DA according to the manufacturer's protocol. The fluorescence intensity of the DCF product was determined using a SpectraMax GEMINI XS fluorescence spectrophotometer (Molecular Devices) at an excitation of $485 \mathrm{~nm}$ and an emission of $538 \mathrm{~nm}$.

NADPH oxidase (NOX) activity was determined from the oxidation of dihydroethidium to ethidium by measuring the superoxide anion formed (32). Cells were incubated with 0.2 $\mathrm{mmol} / \mathrm{L} \mathrm{NADPH}$ as substrate, $0.5 \mathrm{mg} / \mathrm{ml}$ salmon testis DNA, and $0.02 \mathrm{mmol} / \mathrm{L}$ dihydroethidium. Ethidium-DNA fluorescence was measured over $30 \mathrm{~min}$ at excitation and emission wavelengths of $480 \mathrm{~nm}$ and $610 \mathrm{~nm}$, respectively. NOX was expressed as the change in ethidium fluorescence per $\mathrm{mg}$ per hour.

\section{Western blotting}

Crude extracts were mixed with sodium dodecyl sulfatepolyacrylamide gel electrophoresis (SDS-PAGE) loading buffer, boiled for $5 \mathrm{~min}$, and analyzed with 12\% SDS-PAGE. Equal amounts of proteins were loaded into $12 \%$ SDS-PAGE, and resolved proteins were transferred to a nitrocellulose membrane. Immunoreactive bands of GSK-3 $\beta$, p-GSK-3 $\beta$, Akt, p-Akt, p-38, p-p38, NF-kB p65, and $\beta$-actin in the crude extracts were detected and analyzed using an enhanced chemiluminescence kit according to the manufacturer's instructions (Amersham Bioscience, Piscataway, NJ, USA).

\section{Statistical analysis}

All data are the mean \pm SD of 3 independent experiments. Individual differences between the groups were analyzed using one-way analysis of variance. The Student's $t$ test was used to analyze the differences between two groups, and $\mathrm{P}<$ 0.01 was considered to indicate statistical significance.

\section{ACKNOWLEDGEMENTS}

This study was supported by the Basic Science Research Program through the National Research Foundation of Korea (NRF) funded by the Ministry of Education (2015R1D1A1A0 9056947 and 2015R1D1A3A01015793) and by a Student Research Grant from the University of Ulsan College of Medicine, Seoul, Korea.

\section{CONFLICTS OF INTEREST}

The authors have no conflicting interests.

\section{REFERENCES}

1. Cerpa W, Dinamarca MC and Inestrosa NC (2008) Structure-function implications in Alzheimer's disease: effect of Abeta oligomers at central synapses, Curr Alzheimer Res 5, 233-243

2. Li J, Yang JY, Yao XC et al (2013) Oligomeric A $\beta$-induced microglial activation is possibly mediated by NADPH oxidase. Neurochem Res 38, 443-452

3. Guo Y, Shi S, Tang M et al (2014) The suppressive effects of gx-50 on A $\beta$-induced chemotactic migration of microglia. Int Immunopharmacol 19, 283-289

4. Mosher KI and Wyss-Coray T (2014) Microglial dysfunction in brain aging and Alzheimer's disease. Biochem Pharmacol 88, 594-604

5. Krementsov DN, Thornton TM, Teuscher $C$ and RinconM (2013) The emerging role of p38 mitogen-activated protein kinase in multiple sclerosis and its models. Mol Cell Biol 33, 3728-3734

6. Lowe JT, Lee MD, Akella LB et al (2012) Synthesis and profiling of a diverse collection of azetidine-based scaffolds for the development of CNS-focused lead-like libraries. J Org Chem 77, 7187-7211

7. Dalla Y, Singh N, Jaggi AS, Singh D and Ghulati P (2009) Potential of ezetimibe in memory deficits associated with dementia of Alzheimer's type in mice. Indian J Pharmacol $41,262-267$

8. Han M, Song C, Jeong N and Hahn HG (2014). Exploration of 3-aminoazetidines as triple reuptake inhibitors by bioisosteric modification of 3-aoxyazetidine. ACS Med Chem Lett 5, 999-1004 
9. Yun J, Han M, Song C, Cheon SH, Choi K and Hahn HG (2014) Synthesis and biological evaluation of 3-phenethylazetidine derivatives as triple reuptake inhibitors. Bioorg Med Chem Lett 24, 3234-3237

10. Kim EA, Cho CH, Kim J et al (2015) The azetidine derivative, KHG26792 protects against ATP-induced activation of NFAT and MAPK pathways through P2X7 receptor in microglia. Neurotoxicology 51, 198-206

11. Kim J, Kim SM, Na JM, Hahn HG, Cho SW and Yang SJ (2016) Protective effect of 3-(naphthalen-2-yl(propoxy) methyl)azetidine hydrochloride on hypoxia-induced toxicity by suppressing microglial activation in BV-2 cells. BMB Rep 49, 687-692

12. Ebert S, Gerber J, Bader S et al (2005) Dose-dependent activation of microglial cells by Toll-like receptor agonists alone and in combination. J Neuroimmunol 159, 87-96

13. Bach JP, Mengel D, Wahle T et al (2011) The role of $\mathrm{CNI}-1493$ in the function of primary microglia with respect to amyloid-beta. J Alzheimers Dis 26, 69-80

14. Shahani N and Sawa A (2011) Nitric oxide signaling and nitrosative stress in neurons: role for S-nitrosylation. Antioxid Redox Signal 14, 1493-1504

15. Tayler H, Fraser T, Miners JS, Kehoe PG and Love S (2010) Oxidative balance in Alzheimer's disease: Relationship to APOE, Braak tangle stage, and the concentrations of soluble and insoluble amyloid- $\beta$. J Alzheimers Dis 22, 1363-1373

16. Butterfield DA and Lauderback CM (2002) Lipid peroxidation and protein oxidation in Alzheimer's disease brain: potential causes and consequences involving amyloid beta-peptide associated free radical oxidative stress. Free Radic Biol Med 32, 1050-1060

17. Sultana R, Ravagna A, Mohmmad-Abdul H, Calabrese V and Butterfield DA (2005) Ferulic acid ethyl ester protects neurons against amyloid beta-peptide(1-42)-induced oxidative stress and neurotoxicity: relationship to antioxidant activity. J Neurochem 92, 749-758

18. Qureshi GA, Baig S, Sarwar M and Parvez SH (2004) Neurotoxicity, oxidative stress and cerebrovascular disorders. Neurotoxicology 25, 121-138

19. Chen JX and Yan SS (2010) Role of mitochondrial amyloid-beta in Alzheimer's disease. J Alzheimers Dis 20, S569-S578

20. Part K, Kunnis-Beres K, Poska H, Land T, Shimmo R and Fernaeus SZ (2015) Amyloid $\beta_{25-35}$ induced ROS-burst through NADPH oxidase is sensitive to iron chelation in microglial Bv2 cells. Brain Res 1629, 282-290

21. Yao $\mathrm{Y}, \mathrm{Li}$ J, Niu $\mathrm{Y}$ et al (2015) Resveratrol inhibits oligomeric A $\beta$-induced microglial activation via NADPH oxidase. Mol Med Rep 12, 6133-6139

22. Hernandez F, Lucas JJ and Avila J (2013) GSK3 and tau: two convergence points in Alzheimer's disease. J Alzheimers Dis 33, S141-S144

23. Zou Y, Hong B, Fan L et al (2013) Protective effect of puerarin against beta-amyloid-induced oxidative stress in neuronal cultures from rat hippocampus: involvement of the GSK-3ß/Nrf2 signaling pathway. Free Radic Res 47, 55-63

24. Dobarro M, Gerenu G and Ramirez MJ (2013) Propranolol reduces cognitive deficits, amyloid and tau pathology in Alzheimer's transgenic mice. Int J Neuropsychopharmacol 16, 2245-2257

25. Terai K, Matsuo A and McGeer PL (1996) Enhancement of immunoreactivity for NF-kappaB in the hippocampal formation and cerebral cortex of Alzheimer's disease. Brain Res 735, 159-168

26. Zhang J, Zhen YF, Pu-Bu-Ci-Ren et al (2013) Salidroside attenuates beta amyloid-induced cognitive deficits via modulating oxidative stress and inflammatory mediators in rat hippocampus. Behav Brain Res 244, 70-81

27. Zhu X, Rottkamp CA, Hartzler A et al $(2001)$ Activation of MKK6, an upstream activator of $\mathrm{p38}$, in Alzheimer's disease. J Neurochem 79, 311-318

28. Han Y, Han M, Shin D, Song C and Hahn HG (2012) Exploration of novel 3-substituted azetidine derivatives as triple reuptake inhibitors. J Med Chem 55, 8188-8192

29. Kim SJ, Cha JY, Kang HS et al (2016) Corosolic acid ameliorates acute inflammation through inhibition of IRAK-1 phosphorylation in macrophages. BMB Rep 49, 276-281

30. Reznick AZ and Packer L (1994) Oxidative damage to proteins: spectrophotometric method for carbonyl assay. Methods Enzymol 233, 357-363

31. Lauderback CM, Hackett JM, Huang FF et al (2001) The glial glutamate transporter, GLT-1, is oxidatively modified by 4-hydroxy-2-nonenal in the Alzheimer's disease brain: the role of amyloid beta (1-42). J Neurochem 78, 413-416

32. Li MH, Jang JH, Sun B and Surh YJ (2004) Protective effects of oligomers of grape seed polyphenols against beta-amyloid induced oxidative cell death. Ann NY Acad Sci $1030,317-329$ 